Urologe 2022 $61: 304$

https://doi.org/10.1007/s00120-021-01638-x

Online publiziert: 7. September 2021

(c) Springer Medizin Verlag GmbH, ein Teil von

Springer Nature 2021

\section{Erratum zu: Zur Praxis der Therapieentscheidung beim lokal begrenzten Prostatakarzinom: Operation vs. Bestrahlung - wer profitiert?}

\author{
Allokation und Ergebnisse einer unizentrischen, \\ kumulativen Langzeitstudie
}

\author{
W.-D. U. Böhm - R. Koch ${ }^{2}$ - S. Latarius ${ }^{4}$ - A. Mehnert ${ }^{3}$ - C. Werner ${ }^{3} \cdot$ Manfred P. Wirth $^{4}$ \\ 'Akademische Lehrpraxis für Urologie am Universitätsklinikum "Carl Gustav Carus", Technische Universität \\ Dresden, Dresden, Deutschland \\ ${ }^{2}$ Institut für Biometrie und Medizinische Statistik, Universitätsklinikum „Carl Gustav Carus", Technische \\ Universität Dresden, Dresden, Deutschland \\ ${ }^{3}$ Klinisches Krebsregister Dresden, Dresden, Deutschland \\ ${ }^{4}$ Klinik und Poliklinik für Urologie, Universitätsklinikum "Carl Gustav Carus", Technische Universität \\ Dresden, Dresden, Deutschland
}

\section{Erratum zu:}

Urologe 2021

https://doi.org/10.1007/s00120-021-01601-

w

In der zunächst online publizierten Version dieses Artikels sind Fehler in der Zusammenfassung und der Schreibweise eines Autorennamens aufgetreten. Der Beitrag wurde korrigiert und wir bitten Sie, die korrekte Version zu beachten.

\section{Korrespondenzadresse}

\section{Dr. med. W.-D. U. Böhm}

Akademische Lehrpraxis für Urologie am Universitätsklinikum "Carl Gustav Carus", Technische Universität Dresden

Georg-Nerlich-Str. 2, 01307 Dresden, Deutschland

dr.wolf-diether.boehm@gmx.de
Die Online-Version des Originalartikels ist unter https://doi.org/10.1007/s00120-021-01601-w zu finden.

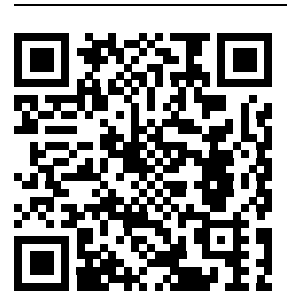

QR-Code scannen \& Beitrag online lesen 